$611-39$

\title{
impresiones de una visita a la industria del cemento norteamericana *
}

\author{
JOSE M. ${ }^{a}$ DE ARTEAGA LARUMBE \\ Subdirector de eLa Auxiliar de la Construcción, S. A.w
}

E1 Instituto de la Construcción y del Cemento me encarga les dirija una breve charla de media hora, como representante más autorizado de un grupo de directores y téc nicos que en noviembre pasado visitó los Estados Unidos, sin que la representación que me honro en ostentar se deba a algún mérito, sino, sencillamente, a ser el de más edad. Varios de mis compañeros de viaje están en la sala, y al finai de este resumen tendremos mucho gusto en tratar de contestar todos a cuantas preguntas quieran dirigirnos.

Los Estados Unidos han producido el año 1955 unos cincuenta millones de toreladas de cemento, es decir, más de trescientos quince $\mathrm{kg}$ de cemento por habitante $\mathrm{y}$ año: el más alto consumo específico del mundo.

Pero entiendo que para poder comprender la organización y forma de trabajar de esta poderosa industria, es preciso formarsc una idea del ser que la ha creado y desarrollado: del norteamericano.

El ciudadano de este pais es el resultado de una mezcla no intima todavia o de una presencia, por llamarlo así, de razas, credos e idiosincrasias en su mayor parte de origen sajón, aunque los latinos de origen son una minoría muy notable, además de eslavos,

(*) Fonencia presentadi en los 1 I Caloquios de Directores y Técnicos de Fúbricas de Cemento. I. T. C. C., marzo 1907. chinos, japoneses y negros de Africa, manumitidos desde solamente tres generaciones. La mayor parte proceden, pues, de personas completamente libres de los viejos prejuicios europeos, y cuyo problemi fundamental al liegar al Nuevo Mundo era el de subsistir. Para ello, y enfrentándose con grandes posibilidades y grandes fenómenos de la Naturaieza (de todos es conocida la violencia de los huracanes y las abrumadoras crecidas de sus gigantescos ríos), el explorador, el pionero, no solamente tuvo que realizar un esfuerzo sostenido durante muchos af́os, desarroliando su ingenio para improvisar soluciones, sino también llegó a la rápida conclusión de que, solamente aunando esfuerzos bien dirigidos, podia tener éxito. Muchos fracasos en cualquier otro sentido lo demostraron ampliamente. He aquí, pues, el origen, el germen de las características del norteamericano de hoy dia: una gran conciencia profesional, derivada de una buena utilidad de su trabajo para el grupo, y un sentido muy desarrollado del trabajo en equipo, de la colaboración entre los componentes de una empresa. Naturalmente, este sentido de colaboración no se podía mantener sin un respeto profundo por los valores humanos individuales, con su consigtriente secuela de respeto a las costumbres, a las crencias religiosas, etc., al mismo tiempo que se hizo evidente la necesidad de una inteligente dirección que concatenase los esfuerzos, lo 
que derivó, en varias generacionès, a la acep. tación de una fuerte autoridad, apoyada por la mayotía.

Pero, además, los recursos naturales de este pais, verdadero campo de experiencias de todos los ordenes para la Humauidad, fueron tales en magnitud y de resultados tan óptimos, que el norteamericano, a través de unas pocas generaciones, la adquirido otra cualidad que también lo caracteriza: una gran confianza en sí mismo, en el fa turo de su pueblo; una certeza, casi absolu$\mathrm{ta}, \mathrm{dc}$ lograr lo que como pueblo se propone.

Voy a tratar de hacerles ver cónı estas tres características las veremos brillar en 'a organización, planteamiento, desnrrollo $\mathrm{y}$ expansión de la industria del cemento en los Estados Unidos.

Los cementos fabricados en los Estados Unidos se pueden resumir en cinco tipos fundamentales: un cemento Portland de parecidas características al europeo, aunque de finuras mayores; un cemento de débil calor de hidratación; un cemento resisterte a los sulfatos; un cemento de grandes resistencias iniciales, $y$ un cemento de normal velocidad de fraguado, especial para albañilería. Existen también, aunque en proporciones mity pequeñas, cementos de altos hornos; de escorias; blancos, y uno especial para pozos de petróleo, de fraguado retardado. En varios de ellos se incluye la cuslidad "air-entraining", que los hace especialmente aptos para grandes fríos y para firmes de carreteras.

E1 precio de venta es libre. No obstante, es uniforme en todos los estados de la Unión el precio de 18 dólares la tonelada, excepto para el de albañilería, cuyo precio es de 16 dólares. Los precios de coste son variables y dependen, como es lógico, de la vejez de las instalaciones y de las condiciones locales; pero es un hecho admitido que la economía de mano de obra cle las nuevas instalaciones está compensada por la elevada amortiza- ción, coll lo que hemos sacado la impresión de que viene a oscilar entre 8 y 9 dólares.

El Gobierno Federal tiene un impuesto del 52 por 100 sobre el beneficio resultante, $\mathrm{y}$ las Autoridades estatales y municipales se llevan algo así como un 2 ó un 3 por 100 más, con lo que puede redondearse en un 55 por 100 de los beneficios las cargas tolales por tonelada. E1 saquerío es aparte. Para el cálculo de financiación, $\mathrm{y}$ ello da idea de la solidez de los negocios en esta nación, se prevé como normal una rotación total al año de seis veces el capital móvil. La productividad del capital tiende a medirse por un sistema, llamado aflujo de dinero descontadon, fundado en garantizar un tipo de interés compuesto que amortice el capita! dulrante la vida prevista de la inversión a base de ganancias netas.

Una gran preocupación que hemos encontrado en todas partes ha sido la formacion de directivos de las Empresas. En ellas, las funciones europeas del Presidente del Consejo de Administración son casi desconocidas. El Consejo de Administración se reúne pocas veces al año, eligiendo cada vez un Presidente (Chairman) para llevar poco más que el orden en la discusión de los asuntos, y confía plenamente durante los intervalos en el Gerente (President). Este, asistido de varios vice-gerentes (vicepresidents), lleva sobre sí toda la responsabilidad de la Empresa, tanto en un funcionamiento normal como en un desarrollo futuro; debe tener previstas todas las contingencias de toda índole, $y$ el Consejo de Administración (Board of directors) le hace responsable de cualquier fracaso. Se comprende, pues, la gran preocupación por la formación de directivos, los cuales hemos visto provenir de cualquiera de los especialistas (abogados, contables, ingenieros, químicos, etc.), de nuestro tipo de Empresa, pero que necesitan una formación especial para elevar su punto de vista. A tal efecto, además de cursos especiales en las 
Liniversidades, existe una Asociación de Directores cuya sigla [es A. M. A. (American Wanagement Association)] que trata, mediante seminarios, cursos, folletos, etc., de dar oportunidades a cuantas voluntádes se ofrecen para aumentar conocimientos y am. pliar puntos de vista a los cmpleados de Empresa y a cuantos deseen legitimar uma ambición mediante su esfuerzo personal. A cste respecto, la impresión que inemos obtenido mediante conversaciones con profesores de Universidad y dircctivos, es quiue están considerando la conveniencia de abandonar un poco el típico sistema pragmático sajón para volver hacia la vieja formación en Humanidades (latín y matemáticas), característica de las Universidades de Oxford y Cambridge, heredadas, a su vez, de las Universida. des madres de París, Salamanca y Alcalá de Henares.

Ësta obsesión formativa de individuos alćnza en las Empresas de cemento todos los escalones, y es constante la práctica de cursos de perfeccionamiento, a la que todos tienen acceso.

En cuanto a maquinaria de la industria, no hemos observado nada ntievo, reciente, tanto desde el punto de vista de maquinaria propiamente dicha como desde el de proceso. Ia mayoría de las instalaciones son de vía húmeda, aunque de dimensiones no corrientes en la vieja Europa, sólidamenie proyectadas y ejecutadas. En molienda de cru. do se emplea con notable porcentaje el circuito cerrado con hogares adicionales para tratar materia prima hasta un 3 ó un 4 por 100 de humedad, y en la de clínker empieza at utilizarse el circuito cerrado, aumque todavía en escasa proporción. Nos ha llamado la atención la solidez y amplio đimensicnamiento de los elementos de transporte, $y$, en todas partes, el gran cuidado en la conservación, consecuencia lógica de esa caracteristica de conciencial profesional que citábamos al principio.
Siendo como es nuestra industria de tipo de maquinaria de trabajo permanente (dia $y$ noche), y dependiendo fundamentalmente la rentabilidad del capital invertido en instalaciones, de st estado de conservación, es ver. daderamente notable la forma en que la industria de cemento norteamericana lleva el entretenimiento de su maquinaria. No solamente se lleva un fichero por cada máquina, en el que se consignan las averías, cuándo se repararon y en qué forma, cspecificando lasta la calidad del material empleado en las stustituciones, sino que se prevé con varias semanas de adelanto la labor del personal de entretenimiento; se estudian y archivan aq̣uellas conclusiones a que el tipo de avería da lugar a deducir; se efectúan cursos de adiestramiento periódicos del personal ustario, y se toma nota cuidadosa de los rendimientos obtenidos en relación con el número de averías, para tener en cuenta este factor en la promoción del personal.

Sin tender a una completa automatización, como mucha gente ha podido creerse, es un hecho cierto que en todas partes hemos visto un gran número de controles de todos los tipos; en especial, naturalmente, los eléctricos. $Y$ hemos comentado también entre nosotros, no la abundancia de controles, sino el hecho de que, en todas las factorías que hemos visitado, todos funcionaban. Este fenómeno, desconocido en Europa, pues los componentes del equipo conocemos un gran número de factorías europeas, en el que siempre hemos visto, en mayor o menor proporción, controles afuera de uso» más o menos momentáneamente, es el que más nos ha hecho ver la calidad del obrero norteamericano y su gran sentido de la colaboración, que antes citábamo:, pues dificil es cucontrar un tipo de máquina de control cuyo buen funcionamiento no requiera la conjunción de dos o más especialistas.

En todas las fábricas que hemos visitado estaba perfectantente organizado el sistema 
de repuestos ; partiendo de la base de lia existencia de una gran industria auxiliar que, no solamente proporciona con relativa rapidez los repuestos necesarios, sino que mantiene contacto con el cliente informándose dsi resuitado obtenido con la máquina o clemento suministrado. También le tiene al corriente de las mejoras que sus experiencias van aconsejando, incluso ofertándole pequeños dispositivos que mejoran el rendimiento. EI criterio de sustitución, unánime en todas las Empresas, es el de que un elemento o máquina tiene tha "vidan determinada, $y$ debe sustituirse en cuanto finaliza ese periodo, sin Esperar a que se produzca la rotura o avería; Ios repuestos están clasificados, ordetrados y ubicados en formas ingeniosas $y$ sencillas, dando la impresión en todas parr tes de una eficiencia regular $y$, ayudados por una muy completa instalación de elementos attxiliares (puentes-grúas, polipaston fijos, etcétera), han conseguido que una sustitución de todo a partc de una maquinaria sea problema de un breve período de tiempo. Todos vosotros, que conocéis por desgraciada experiencia los pesos que tenemos que manejar y el brutal trabajo de alguno de nuestros elementos de machaqueo, molienda, etc., os daréis cuenta de la bondad y rentabilidad de estas previsiones.

En cuanto a combustibles, el mayormente utilizado es el carbón. Este se carncteriza por su homogeneidad, con una composición Excelente a base de $7-8$ por 100 de cenizas, 30 a 35 por 100 de volátiles, un poder calorífico de 7.000 a $\mathbf{7 . 5 0 0}$ calorías y un precio, aunque algo alterado por la circunstancia transporte, que oscila alrededor de los 7 dólares tonelada.

El rendimiento térmico de los hornos es francamente bajo; pues, como decía, la mayoría son vía húmeda. Sin embargo, hemos recogido ta preocupación por esta circunstancia, que empieza a dominar, puesto que, al parecer, el Gobierno Federal llamil la aten- ción sobre la posibilidad, no inmediata, pero sí a prever, de que el consumo de carbón actual haga ver el finat de las disponibilidades de este combustible, $y$ en este sentido empiezan a funcionar hornos vía seca, seducidos por el menor consumo, y con instalaciones de recuperación de calor, no por generación de vapor, puesto que el kilovatiocompra sale más barato, sino tipo Lepol o Humboldt.

En este orden de ideas la casa Kennedy van Saun, de Nueva York, ha perfeccionado recientemente un antiguo dispositivo de economía de calor en hornos rotatorios, que parece está dando buen resultado.

El nuevo sistema de hornos verticales con nodulación no ha sido empleado tod:vía en los Estados Unidos, y algunas veces, en nuestras visitas, hemos podido corresponder a la abierta y franca acogida de los técnicos, explicándoles el funcionamiento de ește tipo de horno.

Algo muty notable en lia industria de cemento americano es la existencia de la “Portland Cement Association». Este Organismo, absolutamente no oficial, está compuesto por el 90 por 100 de las Empresas manufactureras de cemento, y su misión es ayudar en la venta a las Empresas bajo el punto de vista técnico, manteniendo la calidad, y desarrollar todas las posibilidades de utilización del producto, ampliando así constantemente el mercado. Para la primera parte dispone de un edificio muy moderno, con laboratorios, salas de cusayos, etc., el el que están previstas todas las posibilidades de pruebas para cementos y hormigones; una biblioteca excelente, puesta completamente al día ; una revistoteca, en la que pueden leerse cuantas publicaciones sobre el hormigón y el cemento se lanzan en el mundo ; un equipo de técnicos, altamente especializados, que edita constantemente folletos sobre la química del cemento, sobre su fabricación, y reuniendo prácticamente datos 
sobre la marcha de las distintas secciones de ias fábricas de sus asociados, pequeños volúmenes sobre la marcha práctica de la molienda, de los hornos, del ensacado, del machaqueo, etc., que sirven de base a sus socios para la formación profesional que antes he citado.

Por otra parte, la Portland Cement Association dispone de representantes en todos los Estados, al menos en número de uno en aquéllos de escaso conisumo de cemento, cuyo trabajo no consiste en hacer propaganda de tal o cual cemento de sus asociaGos, sino, iundamentalmente, en establecer un gran contacto con todos los usuarios para facilitarles gratis instrucciones y enseñanzas sobre el más adecuado empleo del cemento, analizando cuantas anomalías se presentan, comprobando el trabajo de las estructuras proyectadas (todos ellos son técnicos) y enviando en sus informes periódicos, además de una impresión muy vivida de la situación de la construcción en lo que al cemento se refiere, todas las sugerencias e ideas sobre la posible utilización de este producto; es. tos informes, reunidos y publicados por la Asociación, proporcionan a sus socios datos de inestimable valor para sus secciones de ventas. Es dificil imaginar, para una industria, la realización de una ayuda orientadorá más eficiente, idónea $\mathrm{y}$ eficaz que la conseguida por la del cemento en los Estados Unidos con la P. C. A., y ello corrobora, en el más alto grado, ese sentido de la cooperaciós que forma básicamente el carácter del notteamericano que hemos citado.

El obrero norteamericano viene a ganar entre 1,5 y 2,5 dólares por hora; si se tiene en cuenta que para una familia normal bastan unos 4 ó 5 dólares diarios para alimentos, que en cąsa y vestidos necesitan de 2 a 3 dólares diarjos, está expresado el elevado nivel de vida material medio de que goza t.l país.
Los obreros están en su inmensa mayoria sundicados y recientemente se han fundido las dos grandes organizaciones sindicales de la Nación. Pero el Sindicato es totalmente apolítico. La mano de obra está ligada a las Empresas mediante contratos colectivos, rerovables por años y, en general, hemos obtenido la impresión de que el Sindicato constituye una de las más grandes ayudas a las Empresas. En Estados Unidos los dirigentes sindicales no son, en general, esos tipos de hombre demagogos, con un complejo de frustración en sus vidas; esos tipos de viejos obreros lienos de rencor cuyo afán de revancha social parece presidir todos sus actos; sino, frecuentemente, abogados de gran experiencia en lides sociales y mucho más a menudo, maduros directivos de escala inter media que, por conocer perfectamente la industria y haber tratado durante basiantes ấos al personal de toda índole que la mareja, están en muy buenas condiciones para dilucidar cualquier contingencia. Los Sindicatos, pues, defienderz los intereses de su personal asociado; pero, con un gran respeto al valor de las obligaciones que adquiere en sus contratos, vigila cuidadosamente la eficiencia del trabajo contratado. Se preocupa también de toda la organización de retiros $y$, además, y con gran eficacia, de la formación profesional de sus socios, colaborando con las Empresas en cuantas iniciativas se desarrollan en este terreno. Hay que consignar que la cultura media del obrero menos calificado en Estados Unidos viene a ser la equivalente a la del tercer año de bachiller en nuestro país. Es fácil imaginar cómo, partiendo de esa base, se puede disponer de buenos especialistas, si se tiene en cuenta el ambiente de confianza en sí mismo, de conseguirlo todo, que vive un muchacho en los Estados Unidos y las sinnúmeras oportunidades y facilidades que la preocupación por la formación profesional le brinda por todas partes. 
Un aspecto muy importante de la vida laboral en los Estados Únidos es el de la seguridad en el trabajo. Es normal, al entrar a visitar una fábrica, el encontrar de frente una especie de lápida en la que se consigna el número de horas transcurridas sin el menor accidente, $y$ hemos registrado cifras que llegaban a las 8.000 horas. La prevención de accidentes es un capítulo en el que colaboran Empresas, Gobierno y Sindicato, con un tesón y una tan inteligente amplitud, que conducen a estos resultados asombrosos que he citado. Todas las máquinas, sistemas de transporte, conducciones eléctricas, etc., están protegidos bajo ha idea de que sea imposible la producción de accidentes. En los talleres, trazados en el suelo, están marcados los caminos que debe recorrer el visitante $\mathbf{u}$ obrero que se desplaza de un trabajo; cualquier modificación o avería en curso de reparación está organizada de tal forma, que la trayectoria de llegada y salida de piezas, la interrupción de una vía de acceso, la aparición de un hueco que podría dar lugar a una caida, etc., se barrea antes de que se produzca, y ello forma parte de un cuidadoso plan que el equipo de entretenimiento ha debido desarrollat antes de poner manos a la obra. Cualquier indicación comp'ementaria en forma de señales, letreros, avisos, etcétera, que finaliza el conjunto de protecciones, forma parte del plan de trabajos y es lo primero en colocarse. Las Empresas aseguran que desde hace unos ocho o diez años que lleva concediéndose a la seguridad esta importancia, lan notado, en forma progresiva, un aumento en el rendimiento general que llega a cifrarse en $4 \delta 5$ por 100 .

E1 absentismo, ese fenómeno tan europeo y sobre todo latino, por el que un obrero pierde alguna jornada de trabajo sin una causa sólida, es casi desconocido en los Estados Unidos, y ello se debe casi totalmente a la actitud del Sindicato.
En cuanto al mercado, el precio está ba sado en la rnás libre competencia. Esta competencia, aun con thn precio uniforme del cemente, está basada y llevada a cabo por un signo de calidad y de servicio al cliente. Las secciones de ventas de las Empresas resuelven complicados sistemas de transporte en las formas más variadas. Un 50 por 100 del cemento que circula por ferrocarril en los Estados Unidos, to hace enl vagones a grarel, con un tipo casi general de $\mathbf{4 0}$ toneladas de carga, montados en un sistema «truck». En las grandes ciudades, camiones «containers) levan el cemento a grandes centrales fabricantes de hormigón, que hacen llegar camiones eil los que van montadas hormigoneras de gran capacidad, que aprovechan el tiempo de transporte para hacer la mezcla, que liegan cor una precisión de minuto a la obra en construcción y que se vacian automáticamente. En la obra el hormigón se coloca también automáticamente mediante bombas y conductos a presión.

Una solución comercial que nos liamó la atención consiste en un pequeño silo de hortnigón, en la estación de ferrocarril de una ciudad o villa pequeña, con un elevador adosado a la parte que da a una vía muerta. El vagón a granel descarga por gravedad su contenido al pie del elevador y éste ensila el cemento. Una pequeña ensacadora permite al almacenista o vendedor al detalle envasar el cemento en sacos de papel de dos hojas, con lo que el cliente obtiene una notable reducción en el coste, dada la cercanía de su obra.

En cuanto al futuro volumen del mercado, una Disposición aprobada por las Cámaras ha consignado la cifra de 120.000 millones de dólares para un plan de doce años en carreteras. Como dato curioso, puede dar idea de este volumen el hecho de que al presupuesto de conservación exclusivamente para las carreteras del Fistado de California al- 
canza la cifra de $\mathbf{8 0 0}$ millones de dólares, es decir, algo más que todo nuestro presupuesto nacional. El mercado es firme, y el porvenir de la industria cementera parece, con las cifras dadas, tener asegurado un amplio desarrolio.

En general, es muy dificil tratar de dar una impresión exacta sobre la acogida que en todas partes nos ha sido dispensada. Se han satisfecho nuestros menores deseos y curiosidades; se nos han enseñado los libros de control y los detalles más nimios; se ha respondido a nutestras preguntas con una franqueza y claridad sorprendentes; nos han hecho preguntas sobre nuestra industria $y$ nuestro país, en las que latía un deseo de cambiar puntos de vista y una buena fe evidente, acompañada de una cordial curiosidad. $Y$ tanto en su organización comercial como en sus relaciones internas, hemos po dido obtener la segura impresión de que la industria del cemento norteameticana, al tratar por todos los medios de servir y ser útil a sús clientes, está convencida de que se sirve a si misma y contribuye firmemente al mejoramiento y desarrollo de su país. 